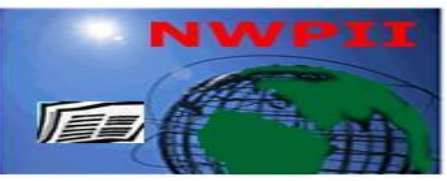

American Journal of Biomedical Sciences

ISSN: 1937-9080

nwpii.com/ajbms

\title{
Evaluation of Dysmorphogenesis in Embryonic Kidney Induced by Calcium Channel Blockers
}

\author{
Nidhi Mahajan", Tarundeep Kaur ${ }^{1}$, Veena Puri ${ }^{2}$, S.K. Singla ${ }^{1}$, Vivekanand Jha ${ }^{3}$, and Sanjeev Puri ${ }^{4 *}$ \\ ${ }^{1}$ Departments of Biochemistry, Panjab University, Chandigarh, India \\ ${ }^{2}$ Centre for systems biology \& Bioinformatics, Panjab University, Chandigarh, India \\ ${ }^{3}$ Department of Nephrology, PGIMER, Chandigarh, India \\ ${ }^{4}$ Department of Biotechnology, UIET and Centre for Stem Cell \& Tissue Engineering, Panjab University, Chandigarh, \\ India \\ *Corresponding Author: \\ Dr. Sanjeev Puri \\ Associate Professor \\ Department of Biotechnology \\ UIET and Centre for Stem Cell \& Tissue Engineering \\ Panjab University \\ Chandigarh-160014, India \\ Tel: 0-91-172-253-4967 \\ Email: spuri_1111@yahoo.co.in
}

Received: 5 November 2011; $\mid$ Revised: 21 December 2011; $\mid$ Accepted: 15 December 2012

\begin{abstract}
Polycystic kidney disease is a common inherited disorder, characterized by formation of multiple fluidfilled cysts in kidney culminating into progressive renal failure. Abnormal proliferation of tubular epithelial cells consequent to genetic mutations associated with PKDl gene together with disruption in calcium $\left(\mathrm{Ca}^{2+}\right)_{\mathrm{i}}$ homeostasis is the hallmark of cystic epithelium. In this regard we sought to exploit microscopy as a tool to assess the ultra-structural alterations both at surface and organelle level. Cystogenesis was induced in mouse metanephroi, (embryonic day, E13.5) using 8-bromocyclic 3'5'-cyclic adenosine monophosphate (8-BrcAMP). Phase contrast microscopy exhibited numerous dilated tubules in metanephroi which continued to enlarge for five days in culture. The effects of 8-Br-cAMP on renal tubular epithelium/cyst epithelium were assessed by histopathological and electron microscopic analysis. Transmission electron microscopy revealed ultra-structural changes like increased vacuolation, swollen and deformed mitochondria, chromatin condensation and disrupted cell membrane in tubular epithelial cells of 8-Br-cAMP treated metanephroi as compared to control. Exposure to $\mathrm{Ca}^{2+}$ channel blockers, nifedipine and gadolinium, further augmented $8-\mathrm{Br}-$ cAMP induced tubular dilations as assessed by histopathological and ultra-structural examinations. Calcium channel brokers augment cAMP induced cystogenesis, possibly through mitochondrial alterations.
\end{abstract}

Keywords: Cysts, 8-Br-cAMP, calcium channel blockers, microscopic alterations. 


\section{Introduction}

The etiology of human congenital renal diseases is best understood through exploring the molecular mechanisms underlying various developmental processes during kidney morphogenesis. Kidney development ensues when the metanephric mesenchyme at the caudal portion of the nephrogenic cord induces the nearby Wolffian duct to produce ureteric bud $[1,2]$. Development proceeds as a result of reciprocal epithelial-mesenchymal signaling between the ureteric bud and metanephric mesenchyme [3, 4]. Any aberration during mesenchymal-epithelial trans-differentiation (MET) would result in the abnormal renal tissue development. Such aberrations are commonly seen in many renal disorders including polycystic kidney disease (PKD) $[5,6]$.

PKD is a chronically progressive genetic disorder affecting approximately one in every 400-1000 live births [7]. The disease is characterized by the growth of numerous renal tubule-derived fluid-filled cysts, which enlarge over a period of time often leading to renal failure [8-11]. Disruption in the functions of $P K D$ gene product have been implicated in cyst formation owing to the inability of tubular epithelial cells to sense mechanical cues that otherwise normally regulate tissue morphogenesis [12-14]. The precise mechanism of cystogenesis, though, still remains largely enigmatic but the role of $\mathrm{Ca}^{2+}$ homeostasis has been imperative $[15,16]$. Numerous reports, in this regards, have also suggested a correlation between altered $\mathrm{Ca}^{2+}$ homeostasis [17] and cAMP [11, 16, 18, 19]. Molecular studies have also suggested that the cAMP pathway(s) promotes both fluid secretion and cell proliferation in PKD renal epithelia. Under the conditions of disrupted $\left(\mathrm{Ca}^{2+}\right)_{\mathrm{i}}$ homeostasis, cAMP mediated pathways are some of the suggestive mechanisms for cyst generation in monolayer culture of renal epithelial cells.

In renal epithelia, the switch in cAMP from an antimitogen to a mitogenic stimulus has been shown to be directly correlated with $\left(\mathrm{Ca}^{2+}\right)$ i levels. Previous studies using $\mathrm{Ca}^{2+}$ channel blockers in M-1 cortical collecting duct cells, human kidney cortex (HKC) cells have shown that abnormal
$\mathrm{Ca}^{2+}$ levels cause a switch to the cAMP growth stimulated phenotype [18]. Most of these studies are focused on the signaling molecules to ascertain the suggestive mechanisms of cystogensis. The reports, however, seems to be lacking on discerning the tissue architectural alterations that may occur in response to suggestive molecules for cystogensis. Hence, we sought to carry out the present study by employing 3-dimensional (3D) metanephric organ culture because the organ culture system permits controlled study of the pathophysiology of cyst formation without vascularization, glomerular filtration, or tubular urine formation. The tubular dilations (cystogenesis) in the metanephric organ culture were microscopically analyzed consequent to 8$\mathrm{Br}$-cAMP treatment in the presence or absence of calcium channel blockers to identify ultrastructural changes at membrane and organelle level. These observations, hence, besides being novel may also provide the basis for therapeutic intervention enroute cystogenesis.

\section{Materials and Methods}

\subsection{Chemicals}

All the chemicals used were of molecular grade and obtained from standard commercial suppliers. Dulbecco's modified eagle medium (DMEM)/ F-12, sodium bicarbonate, penicillinstreptomycin, phosphate buffered saline, HEPES, glutamine, fetal bovine serum (FBS), 8-Br-cAMP, prostaglandin $\mathrm{E}_{1}\left(\mathrm{PGE}_{1}\right)$, insulin, transferrin, selenium, thyroxin, nifedipine, gadolinium hydrochloride were obtained from Sigma chemical company, Saint Louis, (Missouri) U.S.A. For histology and transmission electron microscopy chemicals of analytical grade were used.

\subsection{Animals}

The experimental protocol for the use of animals was approved by the Ethical Committee of Panjab University, Chandigarh, India. Experiments on animals were performed in accordance with the guidelines for the use of laboratory animals approved by the Indian Council of Medical Research, New Delhi, India. 


\subsection{Establishment of metanephric organ culture}

Male and female balb/C mice, weighing 20 30 grams were used for this study. Animals were procured from the Central Animal House of Panjab University, Chandigarh. Males and females were housed separately and were fed with standard pellet diet and water, ad libitum. Timed pregnancies were carried out by crossing male and female mice and the presence of vaginal plugs was used as an indicator for the successful crossing. Negation of any false pregnancy was conceded by monitoring the vaginal smears for estrous cycle arrest in diestrous stage. On embryonic day 13.5 the embryos were dissected out to isolate the metanephroi. These metanephroi were placed on cell culture inserts (BD Pharmingen) and defined culture medium (L-glutamine $2 \mathrm{mM}$, HEPES 10 $\mathrm{mM}$, insulin $5 \mu \mathrm{g} \backslash \mathrm{ml}$, thyroxin $32 \mathrm{pg} \backslash \mathrm{ml}$, selenium $2.8 \mathrm{nM}$, transferrin $5 \mu \mathrm{g} \backslash \mathrm{ml}, \mathrm{PGE}_{1} 25 \mathrm{ng} \backslash \mathrm{ml}$, penicillin $250 \mathrm{U} / \mathrm{ml}$, and streptomycin $250 \mu \mathrm{g} / \mathrm{ml}$ ) was used for their growth.

\subsection{Treatment regimen}

Twenty four hour after the establishment of metanephric organ culture (day 0), the metanephroi were treated with 8-Br-cAMP (100 $\mu \mathrm{M})$ (day1). The generation of cysts were analyzed on the following day (day 2) and continued to microscopically analyze the culture for a period of five days. The effects of different doses of nifedipine and gadolinium $(5 \mu \mathrm{M}$ and 15 $\mu \mathrm{M})$ were studied on these cystic metanephroi starting at day 1. Metanephroi were also treated with dimethyl sulphoxide (DMSO) as vehicle control for 8-Br-cAMP and nifedipine. The progression of the cyst in the embryonic kidneys following drug treatments was monitored periodically by phase contrast microscopy and their effects on the renal tubular epithelium/cyst epithelium were assessed by histopathological and electron microscopic analysis.

\subsection{Histology of cultured metanephroi}

Metanephroi were harvested at $5^{\text {th }}$ day for morphometric analysis. Metanephroi were fixed in formalin followed by dehydration in graded ethanol series. Samples were then embedded in molten paraffin wax and the serial sections of $5 \mu$ thickness using microtome were cut. Sections were deparaffinized in xylene, rehydrated in graded alcohol and stained with haematoxylin and eosin (H \& E). After staining the sections were examined under light microscope (Radical, India) for histopathological analysis.

\subsection{Transmission electron microscopy of cultured metanephroi}

For transmission electron microscopy, metanephroi were fixed in Karnowsky fixative (2.5\% glutaraldehyde and 2\% paraformaldehyde in $0.1 \mathrm{M}$ phosphate buffer, $\mathrm{pH}$ 7.4) overnight. Post-fixation was done in cold $1 \%$ aqueous osmium tetraoxide for 1 hour. After rinsing with phosphate buffer, the specimens were dehydrated in graded ethanol. Metanephroi were processed for embedding using CY 212 Araldite. Ultrathin sections were sliced, stained with uranyl acetate and lead citrate and examined using Morgagni 268D, FEI Company, The Netherlands, transmission electron microscope (AIIMS, New Delhi, India).

\section{Results}

The present study was carried out to evaluate surface and organelle level architectural changes in metanephric organ cultures (E13.5-E18.5) following exposure to 8-Br-cAMP in the absence or presence of $\mathrm{Ca}^{2+}$ channel blockers. No discernable changes in the gross morphology of the metanephroi were observed under defined culture conditions in the presence or absence of different treatments (Fig. 1a-g). The renal explants continued to grow in size and continued the ureteric bud branching and tubule formation over 5 days (Fig. 1a). During all these 5 days, the untreated metanephroi remained healthy showing smooth margins (Fig. 1a) which were affected drastically as seen by diffused margins following cAMP and $\mathrm{Ca}^{2+}$ channel blockers exposure (Fig. 1 $\mathrm{c}-\mathrm{g})$.

Histological analysis of control metanephroi revealed in vitro nephrogenesis as represented by presence of distinct vesicular structures (Fig. 2a) developing into comma and S-shaped bodies eventually destined to become glomeruli (Fig. 3a). Histological examination confirmed presence of few developed glomeruli as well. 


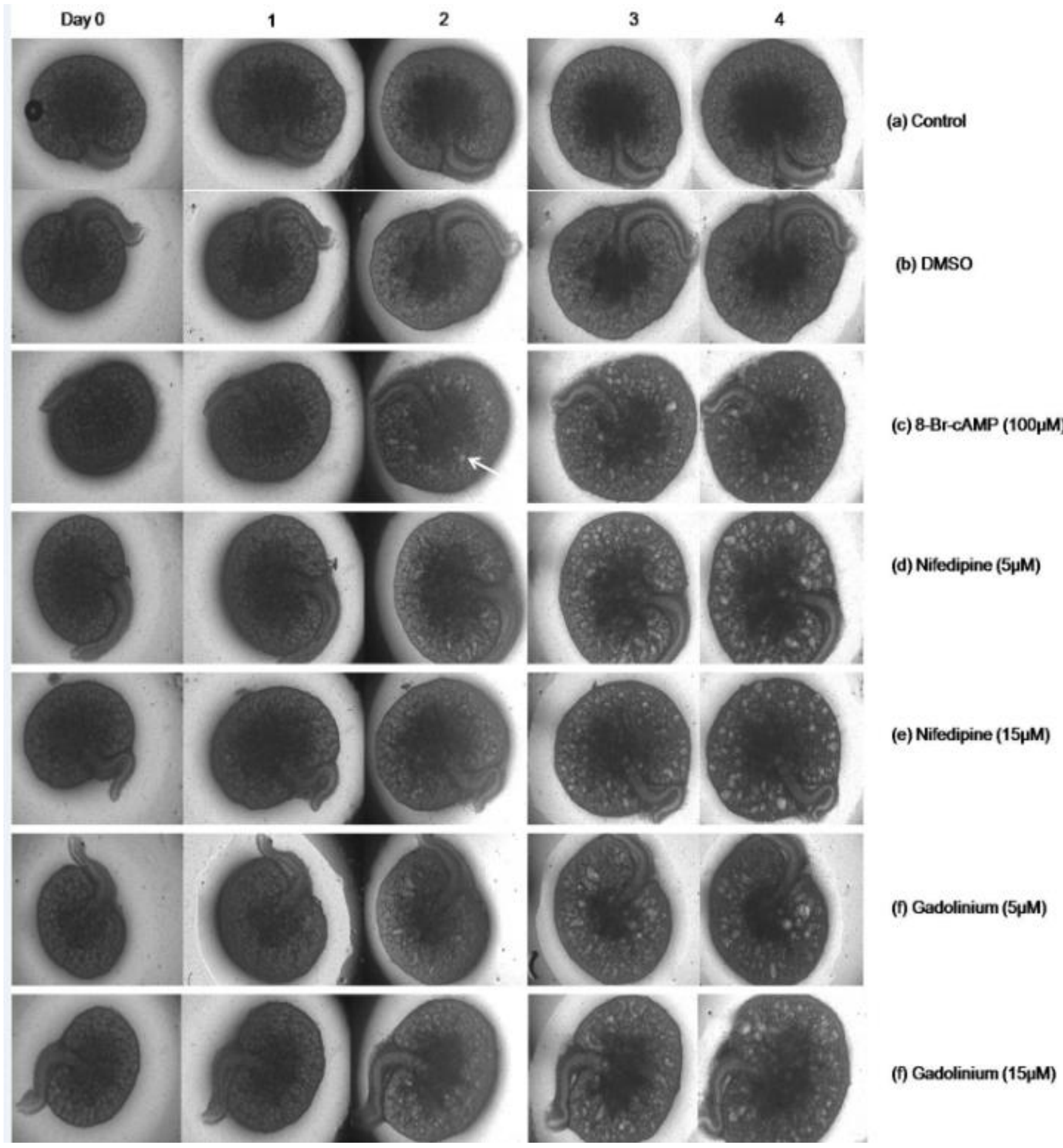

Figure 1. Phase contrast micrographs of control and treated metanephroi (4X) (a) Untreated embryonic kidneys increased in their size along with ureteric bud branching and tubule formation, (b) vehicle treated metanephroi (c) 8 Br-cAMP treatment of embryonic kidneys. Arrow indicates cyst-like structure; small dilations were seen after 24 hour of treatment and continued to enlarge in culture. $(\mathrm{d}, \mathrm{e})$ Nifedipine treatment $(5 \mu \mathrm{M}$ and $15 \mu \mathrm{M}$ respectively) in presence of 8-Br-cAMP enhanced tubular dilations and cyst like structures as compared to 8-Br-cAMP treatment alone (f,g) Gadolinium treatment ( $5 \mu \mathrm{M}$ and $15 \mu \mathrm{M}$ respectively) in presence of 8-Br-cAMP enhanced tubular dilations and cyst like structures as compared to 8-Br-cAMP treatment alone (4X). 


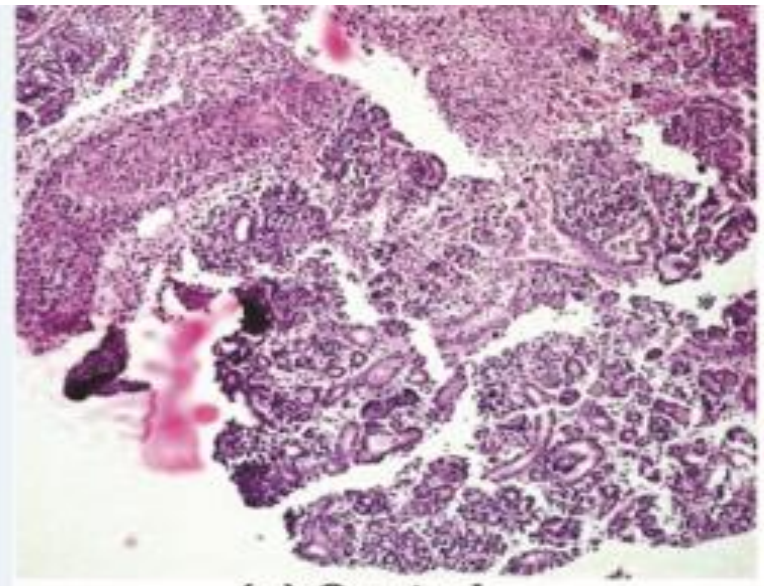

(a) Control

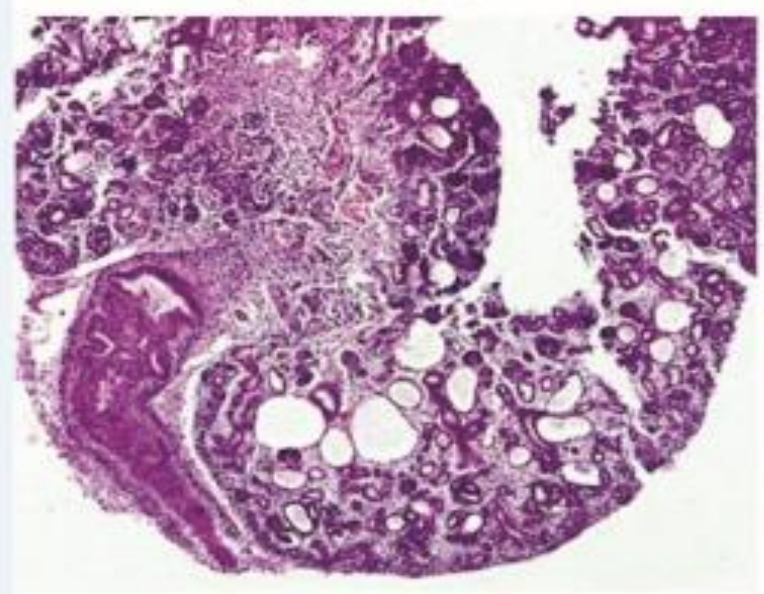

(c) Nifedipine (5 $\mu \mathrm{M})$

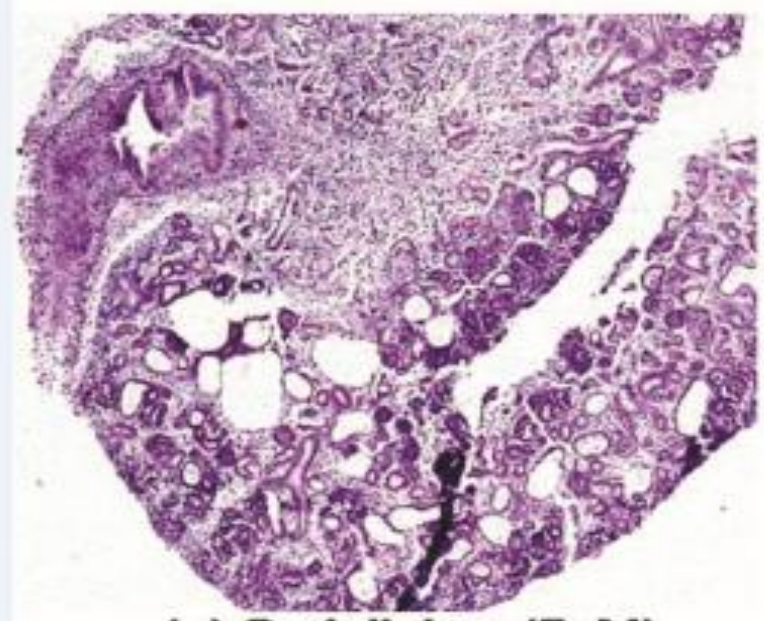

(e) Gadolinium (5 $\mu \mathrm{M})$

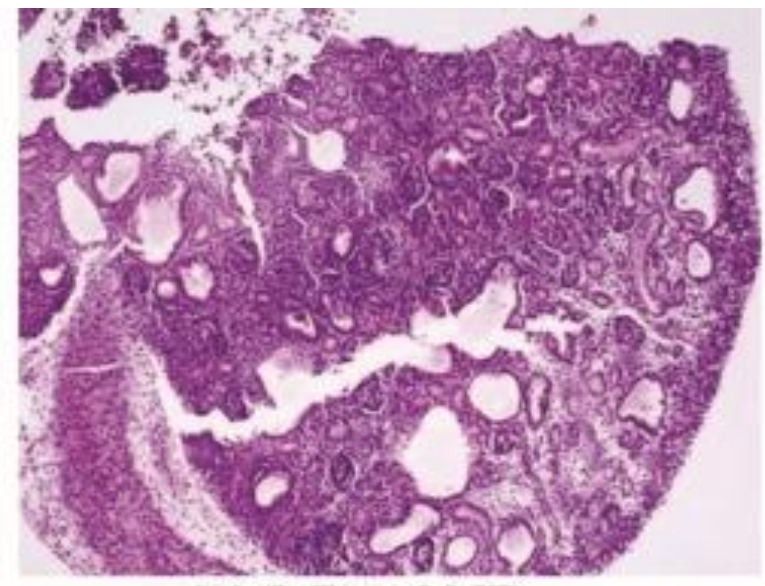

(a) 8-Br-cAMP

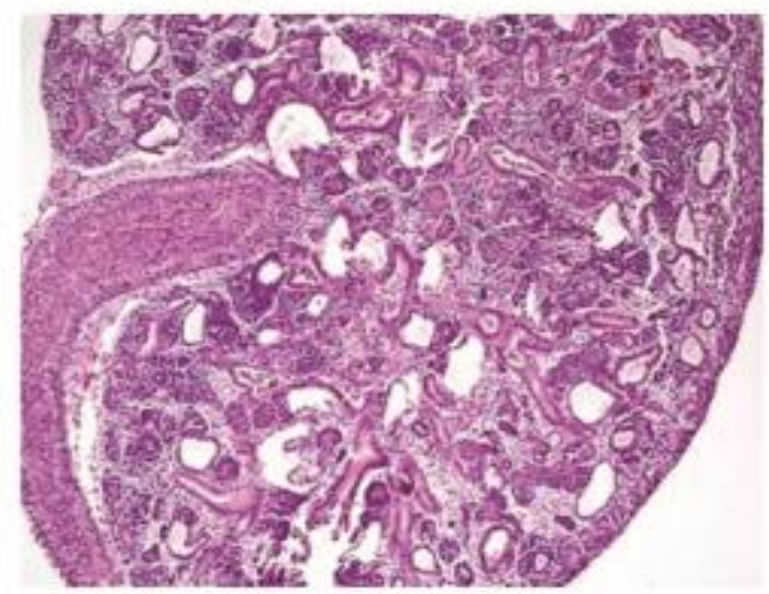

(d) Nifedipine $(15 \mu \mathrm{M})$

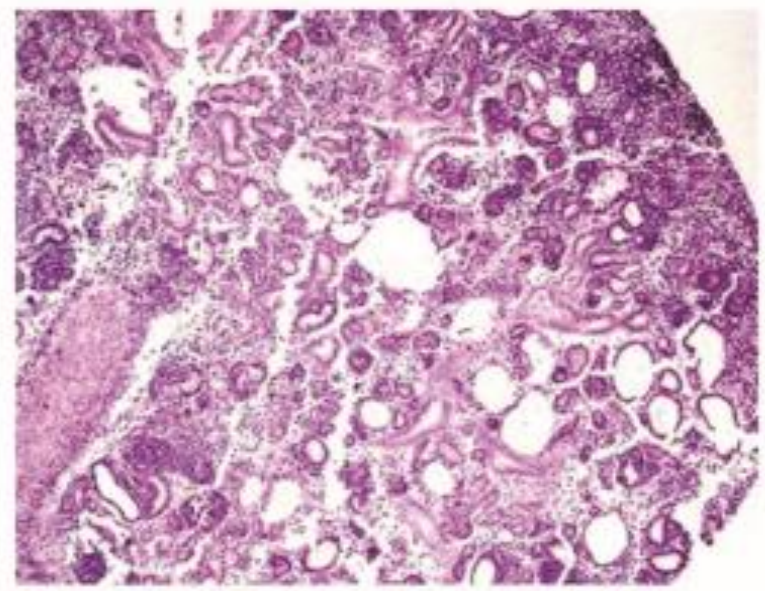

(f) Gadolinium (15 $\mu \mathrm{M})$

Figure 2. Histological micrographs of control and treated metanephroi (20X) (a) normal tubular architecture of untreated metanephroi (b) cyst-like expansions of tubule lumens in 8-Br-cAMP treated metanephroi (c,d) Nifedipine treatment in presence of 8-Br-cAMP increased cyst like structures (e,f) Increased tubular dilations in gadolinium treated metanephroi in presence of $8-\mathrm{Br}-\mathrm{cAMP}(20 \mathrm{X})$. 

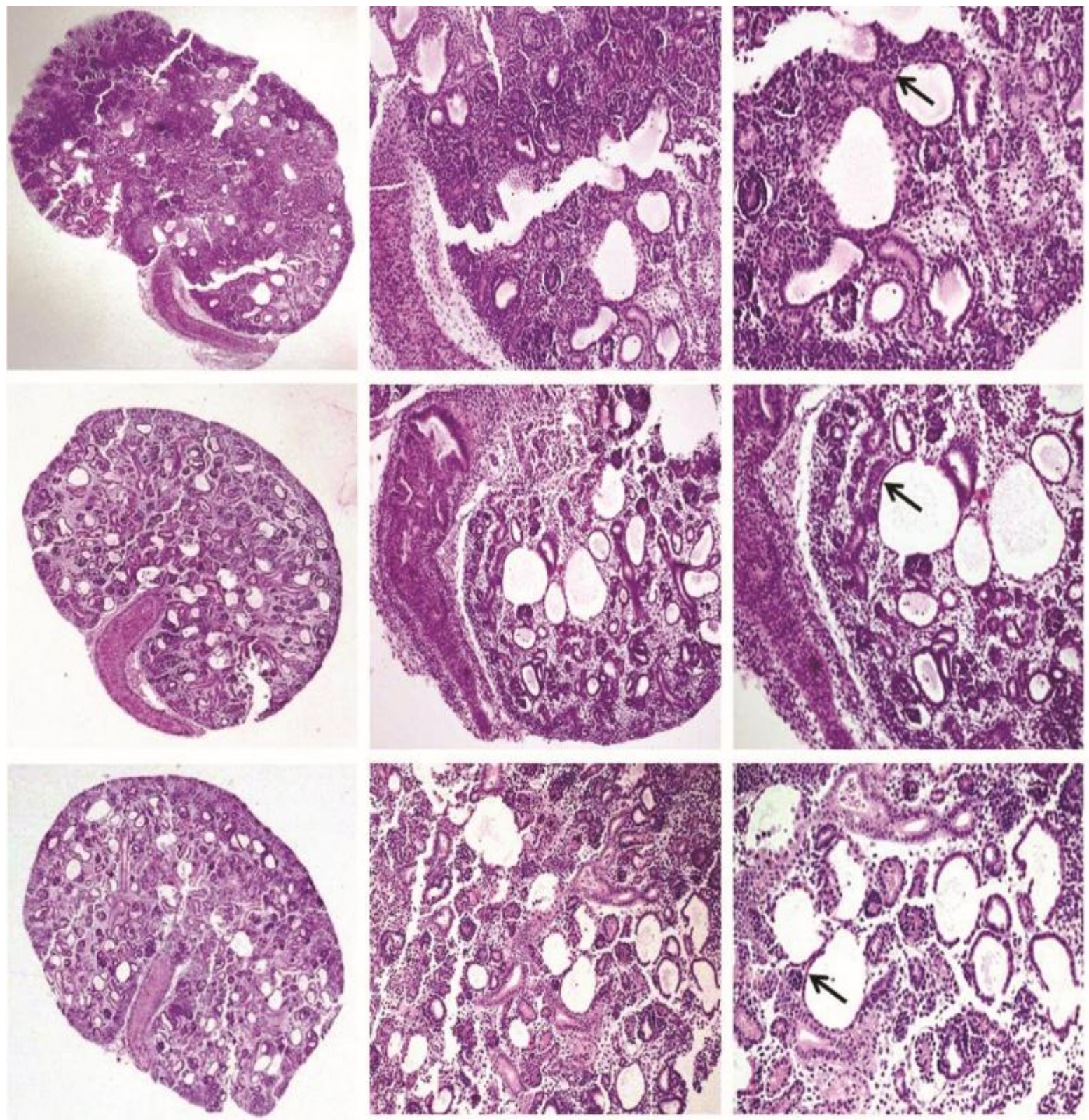

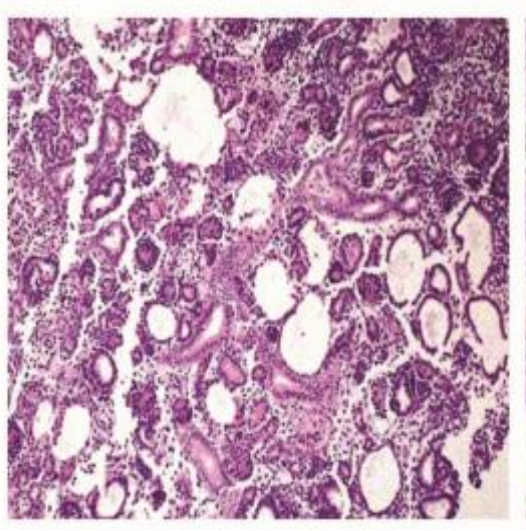

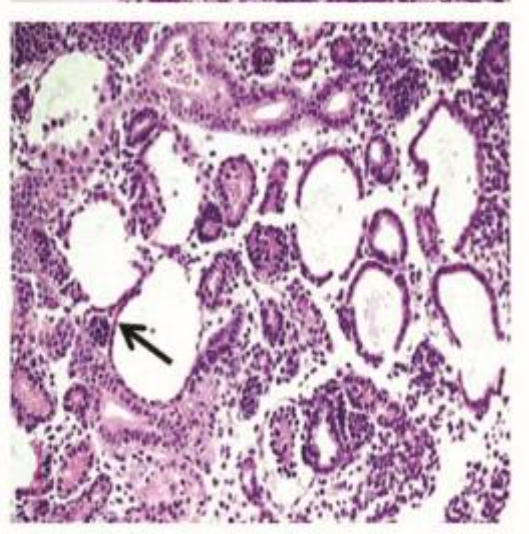

(a) 8-Br-cAMP

(b) Nifedipine

(c) Gadolinium

Figure 3. Light micrographs of cyst-like expansions of tubule lumens (10X, 40X, 100X) Histology showing cystic dilations in cortical and medullary region of metanephroi and thickening of the epithelial lining (arrowheads) of cystic tubules respectively (a) 8-Br-cAMP (b) Nifedipine (c) Gadolinium (10X, 40X, 100X)

The exposure of metanephroi to 8-Br-cAMP treatment is known to produce cystic dilations which were also observed in the present study (Fig. 1c) in comparison to control metanephroi (Fig. 1a,b). These dilations along with membrane distension and puffiness continued to enlarge over a period of 5 days in the presence of 8 -Br-cAMP resulting in expansion into cyst-like structures throughout the metanephroi (Fig. 1c). Representative histology sections of control and 8-
Br-cAMP treated metanephric kidneys confirmed the presence of cyst like tubular dilations (Fig. 2a and $\mathrm{b}$; 3a). Light microscopy of 8-Br-cAMP treated metanephroi depicted developing glomeruli (Fig. 3a). These cAMP triggered tubular dilations were accentuated when the calcium entry to these Br-cAMP organ cultures was inhibited. As shown in Fig. 3c, $\mathrm{Ca}^{2+}$ channel blockers specifically gadolinium treatment led to increase in microcystic dilations in comparison to $8-\mathrm{Br}$ - 
cAMP treatment (Fig 3a). Phase contrast microscopy revealed increase in both micro and few macro level fluidic cystic dilations (Fig. 1d,e,f,g). Histological analysis of gadolinium treated metanephroi revealed cysts structures ranging from large sacs to fusiform dilations (Fig. 3c, 10X). A similar effect, albeit less pronounced, was also observed when another blocker, nifedipine was used (Fig. 3b, 10X). Cells lining the cysts like dilations showed thickened epithelium around all the macrocysts (arrowheads) following treatments with $\mathrm{Ca}^{2+}$ channel blockers (Fig. 3b,c, 100X). The swelling of the tubular epithelia progressed to obliterate the lumen confirming the enhanced cystic phenotype in $\mathrm{Ca}^{2+}$ channel blockers treated metanephroi (Fig. $2 \mathrm{c}, \mathrm{d}, \mathrm{e}, \mathrm{f})$ and reduction in the number of developing and mature glomeruli (Fig. 3b,c). Cystic tubules exhibited a thickened cell wall of flattened epithelial cells as compared to control metanephroi which showed normal tubular epithelial cells (Fig. 3b,c, 40X, 100X).
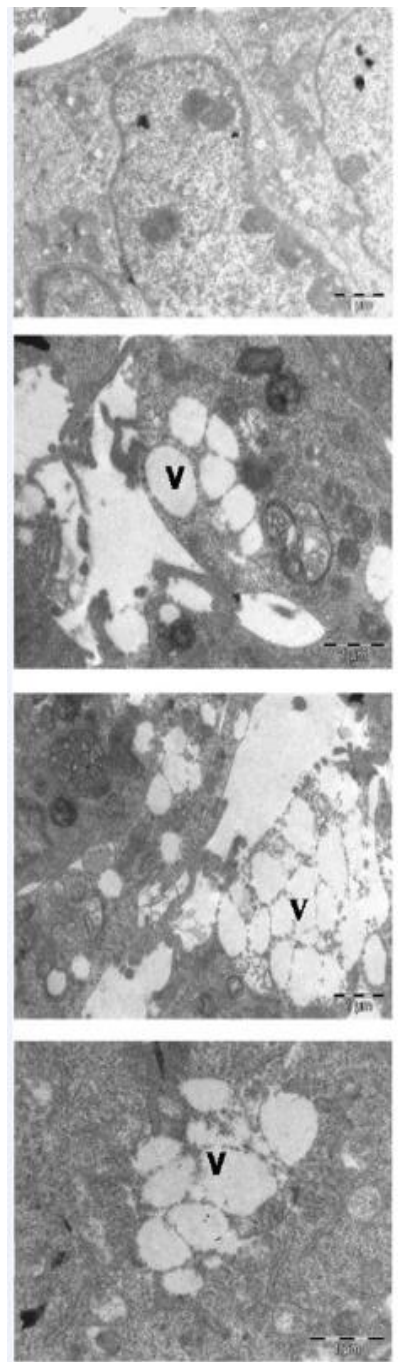

$3200 X$
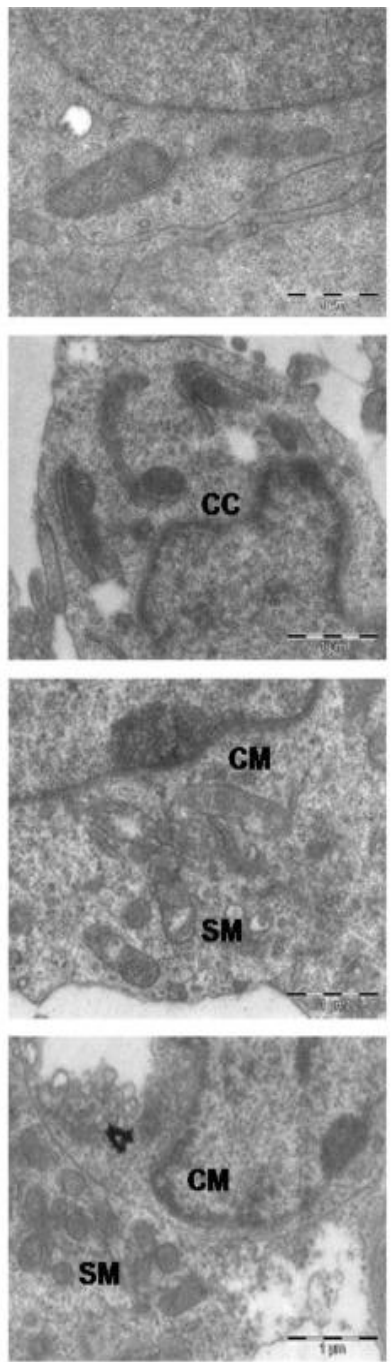

$6300 \mathrm{X}$
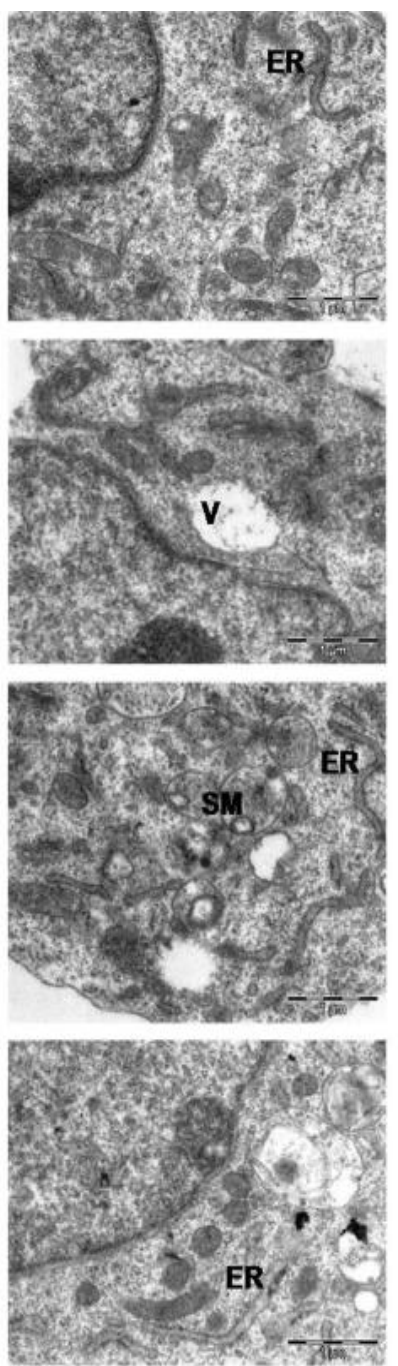

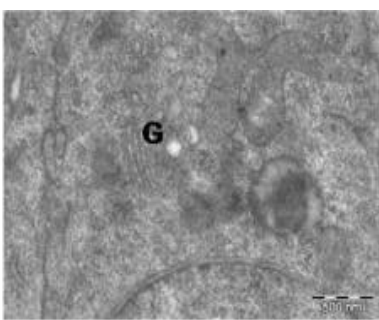

(a) Control

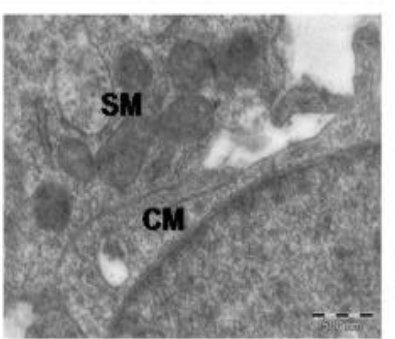

(b) 8-Br-cAMP

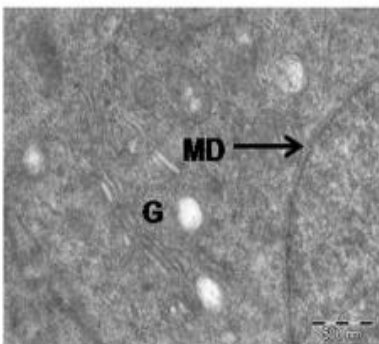

(c) Nifedipne

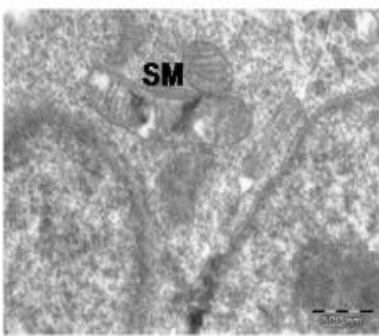

$8000 X$

Figure 4. Transmission electron micrographs of control and treated metanephroi (a) normal tubular epithelial cell (3200X), well defied nuclear envelop, normal mitochondria, golgi apparatus (G) and endoplasmic reticulum (ER) in untreated metanephroi $(6300 \mathrm{X}, 8000 \mathrm{X})$, (b) vacuolation (V, 3200X), chromatin condensation (CC, 6300X), chromatin margination (CM), swollen mitochondria (SM, 8000X) in 8-Br-cAMP treated metanephroi (c) increased vacuolation (3200X), swollen mitochondria, chromatin margination (6300X), dilated endoplsmic reticulum and Golgi bodies, disrupted nuclear membrane $(\mathrm{MD}, 8000 \mathrm{X})$ in nifedipine treatment $(\mathrm{d})$ gadolinium treated increased vacuolation (3200X), swollen mitochondria, dilated endoplasmic reticulum and chromatin margination $(6300 \mathrm{X}, 8000 \mathrm{X})$ in metanephroi. 
Ultrastructural analysis supported the observations made on light microscopic examinations. At cellular level, transmission electron microscopic observations demonstrated aberrations in the structural organization of cell organelle together with marked degenerative changes in few of tubular epithelial cells following 8-Br-cAMP treatment. Multiple foci of swollen and deformed mitochondria and increased vacuolation were also seen. The tubular epithelial cells within cystic metanephroi also demonstrated chromatin condensation, margination, apical blebbing, dilated endoplasmic reticulum and golgi apparatus (Fig. 4b), as compared to control (Fig. 4a).

Further, the effects of nifedipine and gadolinium ( $5 \mu \mathrm{M}$ and $15 \mu \mathrm{M}$ each) were studied on cystogenesis at ultrastructural level in metanephric culture. Electron microscopic observations in metanephroi treated with $\mathrm{Ca}^{2+}$ channel blockers in the presence of 8 -Br-cAMP exacerbated the effects even at organelle level, viz. marked vacuolation in the cytoplasm, chromatin condensation and margination, dilated endoplasmic reticulum and golgi apparatus as compared to 8-Br-cAMP treatment alone. Alterations like mitochondrial swelling, disorientation and shortening of cristae, disruption of nuclear membrane were more pronounced (Fig. $4 \mathrm{c}, \mathrm{d})$, revealing increased cystogenesis in nifedipine and gadolinium treated metanephroi.

\section{Discussion}

By the use of three-dimensional metanephric organ culture, this study provides a comprehensive ultrastructural analysis of tubular dilations and cystic epithelium. Phase contrast microscopic analysis revealed numerous cysts like structures within 24 hour of the administration of $8-\mathrm{Br}$ cAMP which continued to grow in size over a period of 5 days in culture. These tubular dilations seemed to represent the effects of cAMP on fluid secretion, which facilitated the filling up of the fluid in partially differentiated tubular epithelial cells during embryonic development. Accumulation of fluid within dilated tubules represented a cystic morphology in mouse strain C57/BL/6 following 8-Br-cAMP treatment [20].
Increase in the number and volume of microcysts in monolayer cultures of Madin-Darby Canine Kidney Cell (MDCK) and Human Kidney Cortex (HKC) cells following 8-Br-cAMP treatment has also been shown [21, 22]. Renal cAMP levels are elevated in PKD [16]. The stimulatory effect of cAMP on proliferation of cystic epithelial cells derived from human ADPKD kidneys by activating B-RaflMEK/ERK pathway has been deciphered [23]. In contrast, cAMP inhibits cell proliferation in normal human kidney cells and mouse cortical collecting duct cells by inactivating Ras/B-Raf/MEK/ERK pathway [19, 22-25]. Histopathological analysis further validated the presence of numerous tubular fusiform cysts of different sizes distributed both in the cortical and medullary region of 8-Br-cAMP treated metanephroi. These observations draw support from the fact that the cortical epithelial cysts in renal cystic diseases [26, 27], and in ADPKD [2830], demonstrated thickening of epithelial lining, increased vacuolation and narrowing of the lumen due to enlargement of cyst and at ultrastructural level, alterations affecting nucleus, increased cytoplasmic vacuolation, mitochondrial swelling, cristae degeneration, apical blebbing, dilation of organelle [31], were observed. It has also suggested that loss of cristae and indistinct inner mitochondrial membrane were probably due to the cristolysis [28].

In the present study we observed a pattern whereby it seems that $\mathrm{Ca}^{2+}$ channel blockers (nifedipine, L-Type and gadolinium, nonspecific) further augment the 8-Br-cAMP dependent cystic tubular dilations. The reduced number of developing glomeruli and fewer mature nephrons confirmed the role of $\mathrm{Ca}^{2+}$ signaling in nephrogenesis [32]. Further, electron micrographs of metanephroi treated with $\mathrm{Ca}^{2+}$ channel blockers in presence of 8-Br-cAMP, revealed dysmorphogenesis of cell membrane and organelles; and thus, it seems that $\mathrm{Ca}^{2+}$ channel blockers stunted or hampered the standard pace of kidney development and augmented the cystic phenotype formation.

The accentuation of the 8-Br-cAMP effect on mitochondria by $\mathrm{Ca}^{2+}$ channel blockers, suggests that the cystogenesis may probably get additional trigger following leakage of pro-apoptotic signals 
from the mitochondria. Increased apoptosis in PKD may provide a mechanism for the removal of normal parenchyma tissue and cAMP dependent proliferation of cystic epithelia [33-35]. This might be the reason for considerable increase in microcysts, as observed following treatment of 8Br-cAMP treated metanephroi with $\mathrm{Ca}^{2+}$ channel blockers. Moreover, the possibility of a "switch mechanism" [18], on the generation of metanephric cysts can certainly not be ruled out. Observations of the present study whereas lend a support to the switching phenomena, may also provide an additional target to study the cystogenesis. $\mathrm{Ca}^{2+}$ handling by mitochondria is a key feature in cell survival [36-38], and mitochondrial alterations observed after $\mathrm{Ca}^{2+}$ channel blockers treatment may point towards perturbed $\mathrm{Ca}^{2+}$ signaling. It, however, warrants an additional study to delineate the mechanisms that brought in the mitochondrial alterations following different treatments to metanephroi. Blocking $\mathrm{Ca}^{2+}$ supposedly provides loss of polycystin function that, result in dysregulation of $\mathrm{Ca}^{2+}$ signaling, controls renal epithelial cell growth and promotes normal tubular morphogenesis and function [16, 39]. Such observations may thus help in deciphering the potential functions that polycystins play in renal tissue pathophysiology.

In conclusion, the present observations as discerned using electron microscopic examination in cultured embryonic kidneys provide us new insight in understanding cystogenesis at ultrastructural level. Targeting cellular organelles, primarily mitochondria, would pave the way to study human nephrogenesis and pathologies associated with developmental defects.

\section{Acknowledgements}

This research was supported by a grant (Senior Research Fellowship, N.M.) from Council of Scientific and Industrial Research (CSIR) and Department of Biotechnology (DBT) (S.P.), New Delhi, India. The infrastructural and technical support from Center with potential for excellence in biomedical sciences (CPEBS), Panjab University, Chandigarh and Central instrument laboratory (CIL), AIIMS, New Delhi for providing TEM facility, is duly acknowledged.

\section{References}

1. Costantini, F. Renal branching morphogenesis:concepts, questions, and recent advances, Differentiation, 2006, 74, 402-421. DOI: 10.1111/j.1432-0436.2006. 00106.x

2. Yosypiv, I.V. A New Role for the ReninAngiotensin System in the Development of the Ureteric Bud and Renal Collecting System, Keio. J. Med., 2008, 57, 184-189. DOI: $10.2302 / \mathrm{kjm} .57 .184$

3. Meyer, T.N.; Schwesinger, C.; Bush, K.T.; Stuart, R.O.; Rose, D.W.; Shah, M.M.; Vaughn, D.A.; Steer, D.L.; Nigam, S.K. Spatiotemporal regulation of morphogenetic molecules during in vitro branching of the isolated ureteric bud: toward a model of branching through budding in the developing kidney, Dev. Biol., 2004, 275, 44-67. DOI:10.1016/J.YDBIO.2004.07.022

4. Shah, M.M.; Sakurai, H.; Sweeney, D.E.; Gallegos, T.F.; Bush, K.T.; Esko, J.D.; Nigam, S.K. Hs2st mediated kidney mesenchyme induction regulates early ureteric bud branching, Dev. Biol., 2010, 339, 354-365. DOI:10.1016/J.YDBIO.2009.12.033

5. Schedl, A. Renal abnormalities and their developmental origin, Nat. Rev. Genet., 2007, 8, 791-802. DOI: $10.1038 / \mathrm{nrg} 2205$

6. Michos, O. Kidney development: from ureteric bud formation to branching morphogenesis, Curr. Opin. Genet. Dev., 2009,19,484-490.

DOI: $10.1016 /$ j.gde.2009.09.003

7. Belibi, F.A.; Edelstein, C.L. Novel targets for the treatment of autosomal dominant polycystic kidney disease, Expert. Opin. Investig. Drugs, 2010, 19, 315-328. DOI: $\underline{10.1517 / 13543781003588491}$

8. Torres, V.E. Therapies to slow polycystic kidney disease, Nephron Exp. Nephrol., 2004, 98, 1-7. DOI: 10.1159/000079926

9. Al-Bhalal, L.; Akhtar, M. Molecular basis of autosomal dominant polycystic kidney disease, Adv. Anat. Pathol., 2005, 12, 126133. DOI: 10.1097/01.pap.0000163959. 29032.1f 
10. Horie, S. ADPKD: Molecular characterization and quest for treatment. Clin. Exp. Nephrol., 2005, 9, 282-291.

DOI: 10.1007/s10157-005-0367-6

11. Grantham, J.J.; Torres, V.E.; Chapman, A.B.; Guay-Woodford, L.M.; Bae, K.T.; King, B.F.Jr.; Wetzel, L.H.; Baumgarten, D.A.; Kenney, P.J.; Harris, P.C.; Klahr, S.; Bennett, W.M.; Hirschman, G.N.; Meyers, C.M.; Zhang, X.; Zhu, F.; Miller, J.P. Volume progression in polycystic kidney disease, $N$. Engl. J. Med. 2006, 354, 2122-2130.

DOI: 10.1056/NEJMoa054341

12. Ong, A.; Harris, P. Molecular pathogenesis of ADPKD: The polycystic complex gets complex, Kidney Int., 2005, 12, 34-47. DOI: 10.1111/j.1523-1755.2005.00201.x

13. Nauli, S.M.; Alenghat, F.J.; Luo, Y.; Williams, E.; Vassilev, P.; Li, X.; Elia, A.E.; Lu, W.; Brown, E.M.; Quinn, S.J.; Ingber, D.E.; Zhou, J. Polycystins 1 and 2 mediate mechanosensation in the primary cilium of kidney cells, Nat. Genet., 2003, 33, 129-137. DOI: $10.1038 / n g 1076$

14. Nauli, S.M.; Rossetti, S.; Kolb, R.J.; Alenghat, F.J.; Consugar, M.B.; Harris, P.C.; Ingber, D.E.; Loghman-Adham, M.; Zhou, J. Loss of polycystin-1 in human cyst-lining epithelia leads to ciliary dysfunction, J. Am. Soc. Nephrol., 2006, 17, 1015-1025. DOI: 10.1681/ASN.2005080830

15. Puri, S.; Magenheimer, B.S.; Maser, R.L.; Ryan, E.M.; Zien, C.A.; Walker, D.D.; Wallace, D.P.; Hempson, S.J.; Calvet, J.P. Polycystin-1 activates the calcineurin/NFAT (Nuclear Factor of Activated T-cells) signaling pathway, J. Biol. Chem., 2004, 279, 55455-55464.

DOI: 10.1074/jbc.M402905200

16. Wallace, D.P. Cyclic AMP-mediated cyst expansion, Biochim. Biophys. Acta, 2011, 1812, 1291-1300.

DOI: 10.1016/j.bbadis.2010.11.005

17. Somlo, S.; Ehrlich, B. Human disease: Calcium signaling in polycystic kidney disease, Curr. Biol., 2001, 11, 356-360. DOI: 10.1016/S0960-9822(01)00193-2

18. Yamaguchi, T.; Wallace, D.P.; Magenheimer, B.S.; Hempson, S.J.; Grantham, J.J.; Calvet,
J.P. Calcium restriction allows cAMP activation of the B-Raf/ERK pathway, switching cells to a cAMPdependent growthstimulated phenotype. J. Biol. Chem., 2004, 279, 40419-40430.

DOI: 10.1074/jbc.M405079200

19. Yamaguchi, T.; Hempson, S.J.; Reif, G.A.; Hedge, A.M.; Wallace, D.P. Calcium restores a normal proliferation phenotype in human polycystic kidney disease epithelial cells, $J$. Am. Soc. Nephrol., 2006, 17, 178-187. DOI: 10.1681/ASN.2005060645

20. Magenheimer, B.S.; St John, P.L.; Isom, K.S.; Abrahamson, D.R.; De Lisle, R.C.; Wallace, D.P.; Maser, R.L.; Grantham, J.J.; Calvet, J.P. Early embryonic renal tubules of wild-type and polycystic kidney disease kidneys respond to cAMP stimulation with cystic fibrosis transmembrane conductance regulator/ $\mathrm{Na}^{+}, \mathrm{K}^{+}, 2 \mathrm{Cl}^{-}$co-transporterdependent cystic dilation, J. Am. Soc. Nephrol., 2006, 17, 3424-3437. DOI: 10.1681/ASN.2006030295

21. Sullivan, L.P.; Wallace, D.P.; Grantham, J.J. Epithelial Transport in Polycystic Kidney Disease, Physiol. Rev., 1998, 78, 1165-1191.

22. Grantham, J.J.; Ye, M.; Gattone, V.H.II.; Sullivan, L.P. In vitro fluid secretion by epithelium from polycystic kidneys, J. Clin. Invest., 1995, 95, 195-202. DOI: $10.1172 / \mathrm{JCI} 117638$

23. Yamaguchi, T.; Pelling, J.C.; Ramaswamy, N.T.; Eppler, J.W.; Wallace, D.P.; Nagao, S.; Rome, L.A.; Sullivan, L.P.; Grantham, J.J. cAMP stimulates the in vitro proliferation of renal cyst Epithelial cells by activating the extracellular signal-regulated Kinase pathway1, Kidney Int., 2000, 57, 1460-1471. DOI: 10.1046/j.1523-1755.2000.00991.x

24. Calvet, J.P.; Grantham, J.J. The genetics and physiology of polycystic kidney disease, Semin. Nephrol., 2001, 21, 107-123.

DOI: 10.1053/snep.2001.20929

25. Wallace, D.P.; Christensen, M.; Reif, G.; Belibi, F.; Thrasher, B.; Herrell, D.; Grantham, J.J. Electrolyte and fluid secretion by cultured human inner medullary collecting duct cells, Am. J. Physiol. Renal. Physiol., 2002, 283, 1337-1350. 
DOI: 10.1152/ajprenal.00165.2002

26. Filmer, R.B.; Carone, F.A.; Rowland, R.G.; Babcock, J.R. Adrenal CorticosteroidInduced Renal Cystic Disease in the Newborn Hamster, Am. J. Pathol., 1973, 72, 461-472.

27. Avner, E.D.; Sweeney. W.E.; Ellis, D. In vitro modulation of tubular cyst regression in murine polycystic kidney disease, Kidney Int., 1989, 36, 960-968. DOI: 10.1038/ki.1989.288

28. Coleman, M. Multilocular Renal Cyst, Virchows Arch. A. Path. Anat. Histol., 1980, 387, 207-219.

29. Woo, D. Apoptosis and loss of renal tissue in PKD, N. Engl. J. Med., 1995, 333, 18-25. DOI: 10.1056/NEJM199507063330104

30. Morishita, Y.; Matsuzaki, T.; Hara-Chikuma, M.; Andoo, A.; Shimono, M.; Matsuki, A.; Kobayashi, K.; Ikeda, M.; Yamamoto, T.; Verkman, A.; Kusano, E.; Ookawara, S.; Takata, K.; Sasaki, S.; Ishibashi, K. Disruption of Aquaporin-11 Produces Polycystic Kidneys following Vacuolization of the proximal tubule, Mol. Cell. Biol., 2005, 25, 7770-7779.

DOI: $10.1128 / \mathrm{MCB} \cdot 25.17 .7770-7779.2005$

31. Phillips, C.L.; Miller, K.J.; Filson, A.J.; Nurnberger, J.; Clendenon, J.L.; Cook, G.W.; Dunn, K.W.; Overbeek, P.A.; Gattone, V.H.II.; Bacallao, R.L. Renal Cysts of inv/inv Mice Resemble Early Infantile Nephronophthisis, J. Am. Soc. Nephrol., 2004, 15, 1744-1755.

DOI: 10.1097/01.ASN.0000131520.07008.B3

32. Burn, S.F.; Webb, A.; Berry, R.L.; Davies, J.A.; Ferrer-Vaquer, A.; Hadjantonakis, A.K.; Hastie, N.D.; Hohenstein, P. Calcium/NFAT signaling promotes early nephrogenesis, Dev.
Biol., $\quad 2011, \quad 352, \quad 288-298$.

DOI: $\underline{10.1016 / j . y d b i o .2011 .01 .033}$

33. Zhou, X.J.; Kukes, G. Pathogenesis of autosomal dominant polycystic kidney disease: role of apoptosis, Diagn. Mol. Pathol., 1998, 7, 65-68.

DOI: 10.1097/00019606-199804000-00001

34. Murcia, N.S.; Sweeney, Jr.W.E.; Avner, E.D. New insights into the molecular pathophysiology of polycystic kidney disease, Kidney Int., 1999, 55, 1187-1197. DOI:10.1046/j.1523-1755.1999.00370.x

35. Tao, Y.; Kim, J.; Faubel, S.; Wu, J.C.; Falk, S.A.; Schrier, R.W.; Edelstein, C.L. Caspase inhibition reduces tubular apoptosis and proliferation and slows disease progression in polycystic kidney disease (PKD), Proc. Natl. Acad. Sci. USA., 2005, 102, 6954-6959. DOI:10.1073/pnas.0408518102

36. Duchen, M.R. Topical review: Mitochondria and calcium: from cell signaling to cell death, J. Physiol., 2000, 529, 57-68. DOI:10.1111/j.1469-7793.2000.00057.x

37. Rizzuto, R.; Bernardi, P.; Pozzan, T. Topical review: Mitochondria as all round players of the calcium game. J. Physiol., 2000, 529, 3747. DOI:10.1111/j.1469-7793.2000.00037.x

38. Contreras, L.; Drago, I.; Zampese, E.; Pozzan, T. Mitochondria: the calcium connection, Biochim. Biophys. Acta, 2010, 1797, 607618. DOI:10.1016/J.BBABIO.2010.05.005

39. Torres, V.E.; Harris, P.C. Autosomal dominant polycystic kidney disease: the last 3 years, Kidney Int., 2009, 76, 149-168. DOI:10.1038/ki.2009.128 